\title{
Variation of colony morphology and chromosomal rearrangement in Candida tropicalis pK233
}

\author{
TaKahito SuzUKi, ${ }^{*}$ YUki MiYamae and IKUKo Ishida
}

Biological Laboratory, Faculty of Science, Nara Women's University, Nara 630, Japan

(Received 8 June 1990; revised 15 August 1990; accepted 19 September 1990)

\begin{abstract}
UV irradiation treatment of the asexual yeast Candida tropicalis gave rise to morphological mutants exhibiting at least four different types of abnormal colonies on glucose-containing solid medium. These mutants were named according to their colony morphologies: 'doughnut', 'frilly', 'echinoid' and 'walnut' mutants. The doughnut mutant produced a wrinkled colony with a hollow in its central region that was rich in filamentous pseudohyphal cells. With increased incubation time, the colony gradually changed to a reticulate shape. The parent strain, which normally produced smooth colonies, gave similar colonies to those of the doughnut mutant when grown in medium containing oleic acid as carbon source. Both the frilly and the walnut mutants produced pseudohyphal cells in a similar fashion to the doughnut mutant. The echinoid mutant produced an echinulate colony morphology with aerial hyphae and contained true hyphal cells as well as pseudohyphal ones. Pulsed-field gel electrophoresis showed that the echinoid and frilly mutants had different karyotypes from that of their parent strain, suggesting the occurrence of chromosomal rearrangements associated with these morphological mutations.
\end{abstract}

\section{Introduction}

The asexual yeast Candida tropicalis is an organism of both medical and industrial importance. This yeast can assimilate fatty acids or alkanes as the sole source of carbon and energy, and the synthesis of peroxisomes is induced abundantly by growth on these substrates (Kamiryo \& Okazaki, 1984). This yeast is also an opportunistic human pathogen that causes systemic infection (Odds, 1987). The development of genetic systems for this yeast may be valuable in elucidating the induction of peroxisomal synthesis and the mechanisms of pathogenesis.

In the closely related pathogenic yeast, $C$. albicans, variations of colony morphology have been reported, where abnormal colonies arise at a frequency of $10^{-2}$ to $10^{-4}$ (Slutsky et al., 1985; Rikkerink et al., 1988; Suzuki et al., 1989; Rustchenko-Bulgac et al., 1990). In the latter two reports, chromosomal rearrangements were associated with the variation phenomenon.

We report here that variation of colony-morphology phenotypes can be generated in a typical $C$. tropicalis strain, pK233, and that changes in chromosomal DNA banding patterns are correlated with some of the variations.

Abbreviation: PFGE, pulsed-field gel electrophoresis.

\section{Methods}

Organisms, media and growth conditions. C. tropicalis strain pK233 was kindly given to us by $\operatorname{Dr} \mathrm{T}$. Kamihara, Kyoto University, Japan. Saccharomyces cerevisiae strain X2180-1A and C. albicans $\mathrm{FC} 18$ (Whelan et al., 1980) were used as standards for chromosomal banding patterns. Yeast cells were grown in YPD liquid medium [containing $1 \%$ (w/v) polypeptone (Wako Pure Chemical Industries), $1 \%(\mathrm{w} / \mathrm{v})$ yeast extract and $2 \%(\mathrm{w} / \mathrm{v})$ glucose] with shaking $\left(100\right.$ strokes $\left.\mathrm{min}^{-1}\right)$ at $30{ }^{\circ} \mathrm{C}$. For observation of colony morphologies, the concentrations of polypeptone, yeast extract and glucose of YPD were reduced to half (1/2YPD) and the medium solidified by adding $2 \%(w / v)$ agar. Agar plates were inoculated with approximately 200 cells of strain pK233 and subjected to $6 \times 10^{-5} \mathrm{~J} \mathrm{~mm}^{-2} \mathrm{UV}$ light from a Toshiba GL $15 \mathrm{~W}$ germicidal lamp. For comparison of colony morphology and peroxisome induction, cells were grown at $30^{\circ} \mathrm{C}$ in YPBO liquid medium and on plates of YPBO medium solidified by adding $2 \%(\mathrm{w} / \mathrm{v})$ agar. The YPBO medium was prepared according to Kamiryo et al. (1981), and contained $0.3 \%$ yeast extract, $0.5 \%$ polypeptone, $0.5 \% \mathrm{~K}_{2} \mathrm{HPO}_{4}, 0.5 \%$ $\mathrm{KH}_{2} \mathrm{PO}_{4}, 1 \%(\mathrm{w} / \mathrm{v}) \mathrm{Brij} 58$ and $1 \%(w / v)$ oleic acid. The control cultures, without proliferation of peroxisomes, were grown in YPBG medium; this was the same as YPBO medium except that the oleic acid was replaced by $2 \%$ glucose.

Microscopy. Colonies were viewed under an Olympus SZH141 stereomicroscope. For observations of cells constituting a colony, cells were picked up with an inoculating needle from various sites of the colony, suspended in a drop of water and observed using a Nikon DIAPHOTO-TMD microscope equipped with Nomarski interferencecontrast optics.

Pulsed-field gel electrophoresis (PFGE). Sample preparation for PFGE was as described previously (Suzuki et al., 1989). After several 
trials, we decided to use a 3 min pulse time at $150 \mathrm{~V}$ for $24 \mathrm{~h}$, followed by a $10 \mathrm{~min}$ pulse time at $110 \mathrm{~V}$ for $24 \mathrm{~h}$, a $20 \mathrm{~min}$ pulse time at $50 \mathrm{~V}$ for $24 \mathrm{~h}$, and finally a $40 \mathrm{~min}$ pulse time at $20 \mathrm{~V}$ for 20 to $40 \mathrm{~h}$, as indicated in the legend to Fig. 3. A $0.9 \%$ agarose (Wako Chemicals) gel $(15 \times 15 \mathrm{~cm})$ was used. The Pulsaphor system (Pharmacia-LKB) equipped with an LKB 2015-100 hexagonal electrode kit was used, with the temperature maintained at $9^{\circ} \mathrm{C}$ by a MultiTemp II thermostatic circulator. Staining and photographing of the gels were according to Carle \& Olson (1984).

DNA determination. Stationary-phase cells were collected by centrifugation, washed and resuspended in $60 \%(\mathrm{v} / \mathrm{v})$ ethanol. A sample was appropriately diluted to count the number of cells microscopically in a haemocytometer. Another sample was used for DNA determination by the method of Burton (1956) according to Stewart (1975), with highly polymerized calf thymus DNA (Sigma) as standard.

\section{Results}

\section{Isolation of colony-morphology mutants}

The parent strain of $C$. tropicalis, pK233, produced smooth colonies on both YPD and 1/2YPD plates (Fig. $1 a$ ). When these smooth colonies were exposed to an oblique light, the surface of the colony was lustrous. Among 9793 UV-surviving colonies from six separate cultures of strain pK233, 75 showed different colonymorphology phenotypes from that of the parent on 1/2YPD plates. Each of these six cultures was derived from a separate smooth colony, thus ensuring that variants arising from separate cultures would be the results of independent events. Single-colony isolations were repeated on each of these variant colonies, and stabilities and characteristic morphologies for these 75 variants were determined both after incubating for 2 weeks at room temperature and for $5 \mathrm{~d}$ at $30^{\circ} \mathrm{C}$. More than $95 \%$ of the colonies of each variant were stable during several successive subcultures. These variants are hereafter called 'colony-morphology mutants'; they were grouped into four classes, as follows.

The first class, composed of 32 mutant isolates, was of the irregular wrinkled type (Fig. 1c), apparently similar to the variants described by Slutsky et al. (1984) in $C$. albicans. The shape of the colony of this type was initially like a doughnut, the central region having a depression. During prolonged incubation, its surface altered, becoming irregular-wrinkled. We will refer to this type as doughnut mutants. The second class, including four isolates, was characterized by the presence of a smooth central region and peripheral frills on the surface of colonies (Fig. 1e). During prolonged incubations, wrinkles developed and enclosed the frills. These are 'frilly' mutants. The third class, consisting of 22 isolates, was of echinulate type with aerial hyphae extending from the surface of colonies (Fig. $1 \mathrm{~g}$ ). A well-separated colony of this type was like a sea urchin in shape. Thus, these mutants are termed 'echinoid'. The surface of the echinulate colony was too hard to scratch off with an inoculating loop. The fourth class, including 17 isolates, had lustreless colonies and the surface of the colonies changed gradually into wrinkled (Fig. 1 $i$ ). Since this resembled the surface of walnuts, we will refer to this type of colony as 'walnut'.

\section{Changes in colony morphologies under peroxisome- inducing conditions}

Kamiryo et al. (1981) showed that peroxisomes were induced when $C$. tropicalis $\mathrm{pK} 233$ was grown aerobically with oleic acid as carbon source. The mutants described above were grown on plates containing medium with oleic acid as carbon source (YPBO medium), and the appearance of colonies was examined. After 1 to $2 \mathrm{~d}$ of culture, when small colonies appeared, the parent strain gave smooth colonies. An interesting finding was the subsequent development of these colonies into wrinkled and doughnut-like ones accompanied by hyphal growth in the agar layer after prolonged culture (over $3 \mathrm{~d}$, Fig. $1 b$ ), although the parent strain had shown the smooth colony-morphology on 1/2YPD plates (Fig. 1a). In striking contrast was the morphology of colonies of the doughnut mutants grown on YPBO: the mutants produced smooth but lustreless colonies (Fig. 1d). Similarly, the frilly mutants also gave smooth but lustreless colonies on YPBO (Fig. $1 f$ ). The echinoid mutants gave rather doughnut-like colonies on this medium and the colonies had fewer aerial hyphae than those grown on 1/2YPD (Fig. $1 h$ ). The walnut mutants showed very weak growth on PYBO; their colony morphology was apparently smooth but lustreless (Fig. $1 j$ ).

In order to examine whether the above changes in colony morphology were due to the difference in the carbon source of the growth media, colonies were also grown on plates of YPBG medium, which contained glucose instead of oleic acid. The morphologies of colonies of the parent and the colony-morphology mutants on YPBG plates were all indistinguishable from those described for each type on $1 / 2$ YPD plates. Therefore, these changes in colony morphologies in the parent strain as well as the mutants can be ascribed to whether glucose or oleic acid was used as the carbon source.

\section{Cytological features of colony-morphology mutants}

Cell morphologies were examined microscopically from colonies grown on $1 / 2 \mathrm{YPD}, \mathrm{YPBO}$ or YPBG plates. Cultures of the parent strain pK233 on both 1/2YPD and 

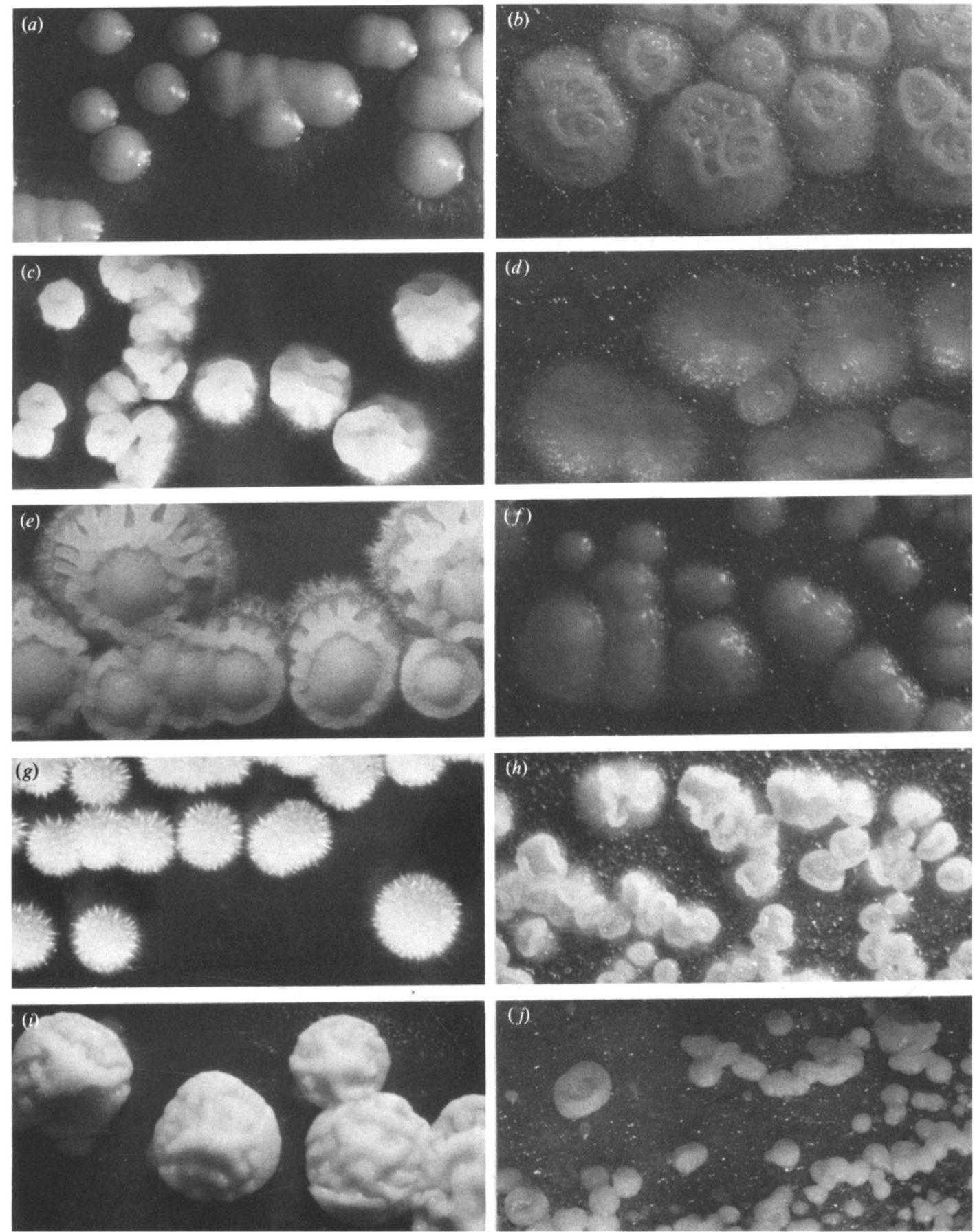

Fig. 1. Colonial morphologies of $C$. tropicalis pK233 $(a, b)$, doughnut mutant D4 $(c, d)$, frilly mutant F2 (e, $f)$, echinoid mutant E49 $(g, h)$, and walnut mutant W7 $(i, j)$. Colonies were grown for $6 \mathrm{~d}$ on 1/2YPD plates $(a, c, e, g, i)$ or on YPBO plates $(b, d, f, h, j)$.

YPBG plates exhibited a uniform cell population of oval or ellipsoid cells having mostly one or no bud (Fig. $2 a$ ). The parent strain also contained a small fraction (less than $1 \%$ ) of pseudohyphal chain-forming cells. Mycelia that developed into the agar layer from the colonies were composed of pseudohyphal cells in chains. In contrast, the colony of the parent strain grown on YPBO plates consisted of pseudohyphal cells as a major fraction and of typical yeast cells at lower frequency (Fig. $2 b$ ). Oil drops were frequently observed on the pseudohyphal cells and large yeast cells containing well-developed vacuoles were present. Wrinkled regions of the colony were rich in pseudohyphal cells forming bundles.

In contrast to the uniform cell population of the parent 

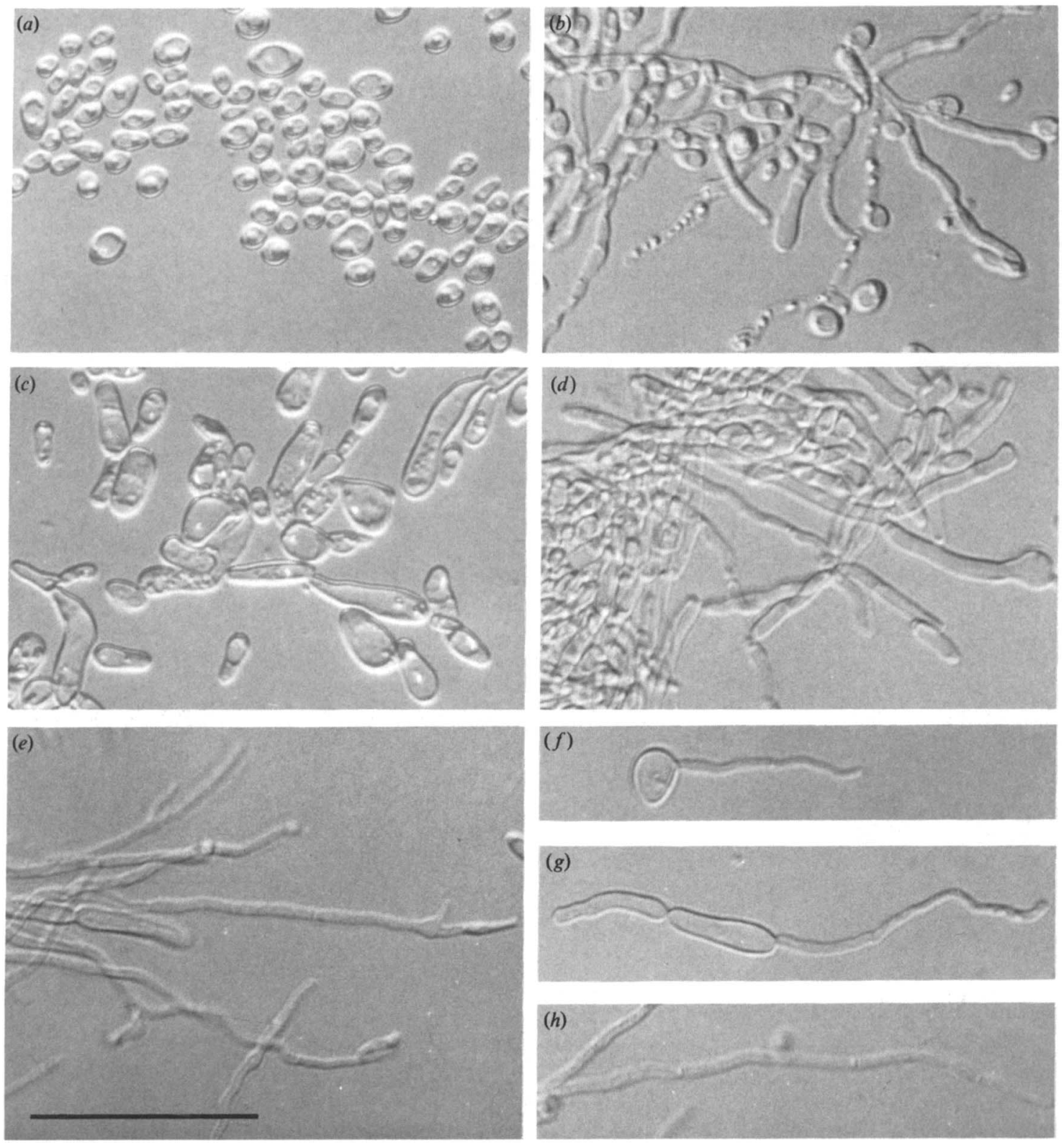

Fig. 2. $(a, b)$ Cell morphologies of $C$. tropicalis pK233 grown on 1/2YPD $(a)$ and on YPBO $(b)$. (c) Walnut mutant W7, (d) doughnut mutant D4, and $(e-h)$ echinoid mutant E49 grown on 1/2YPD. Bar, $40 \mu \mathrm{m}$ (magnification is the same for all the photographs).

strain grown on $1 / 2 \mathrm{YPD}$ plates, the cell population of each colony-morphology mutant consisted of a wide range of different cell sizes and shapes. The walnut mutants, grown on 1/2YPD or YPBG plates, contained obovate to pyriform yeast cells and also elongated ones (Fig. 2c). Pseudohyphal cells in chains, each of which was a thick cylinder with well-developed vacuoles, were also detected as a small percentage of the population. However, on YPBO plates, the growth of the walnut mutants was weak, their colonies were small, wrinkles were rare, and the proportion of chain-forming pseudohyphal cells was much smaller. Therefore, the wrinkled texture of the walnut variants may reflect development of pseudohyphal cells in chains.

Colonies of the doughnut mutants grown on 1/2YPD or YPBG plates consisted of typical pseudohyphal cells, together with normal yeast cells (Fig. $2 d$ ). The terminal cell of a pseudohypha was shorter than the preceding cell behind the first septum. These individual pseudohyphal cells formed filaments more than ten times longer than the yeast cells. In contrast, colonies of the doughnut mutants grown on YPBO plates, which had a smooth texture, were composed of yeast cells similar to those of the parent strain or of elongated yeast cells two to four 


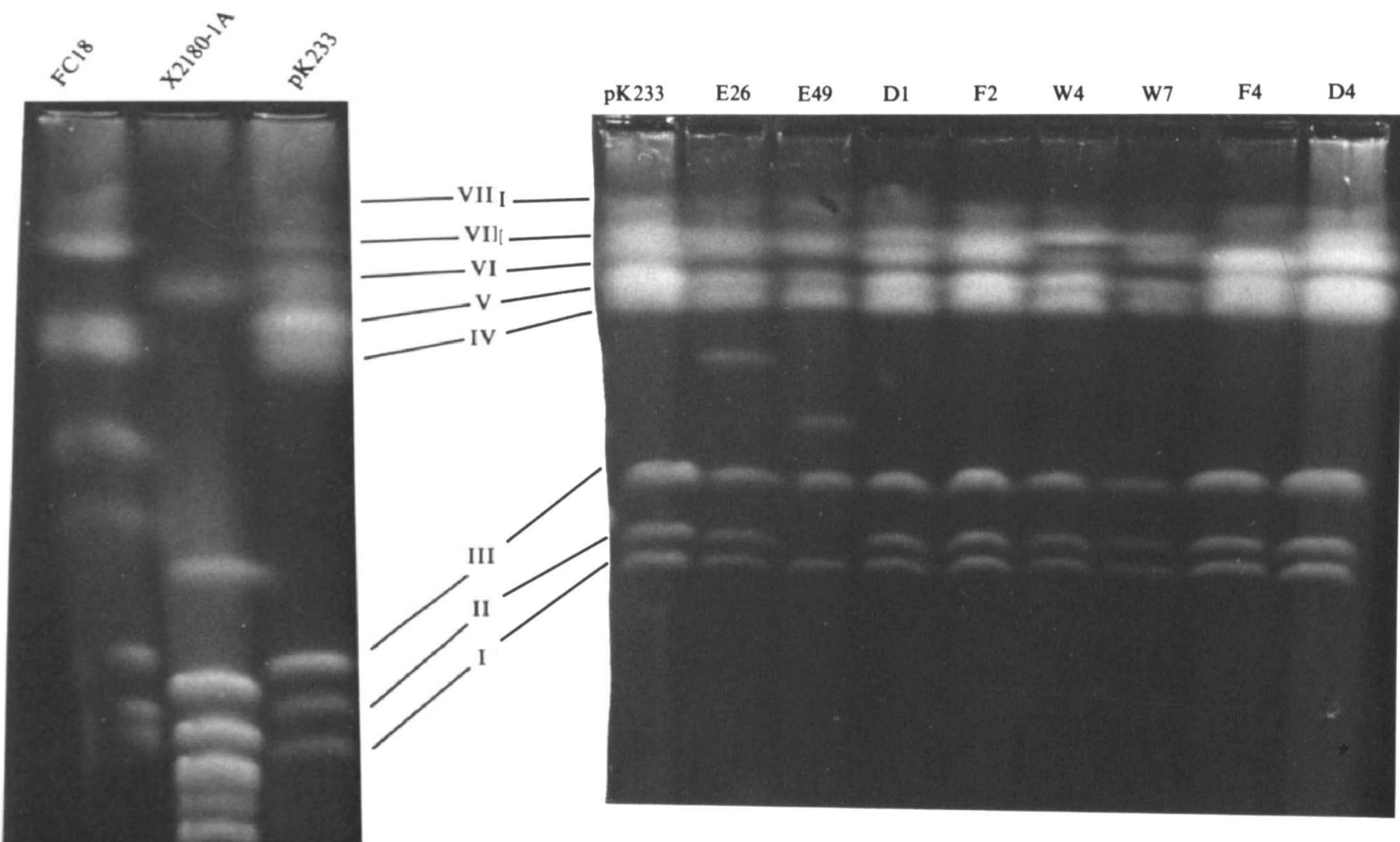

Fig. 3. Separation of $C$. tropicalis chromosomes by PFGE. A $0.9 \%$ agarose gel $(15 \times 15 \mathrm{~cm})$ was run in four sequential steps as described in Methods. The running time of the fourth step was $40 \mathrm{~h}$ (upper left three lanes), or $28 \mathrm{~h}$ (the other lanes). The names of strains are indicated above the lanes. $C$. albicans $\mathrm{FCl} 8$ and $S$. cerevisiae X2180-1 A were used as standards for karyotypes. Echinoid mutants (E1 to E7, E26 and E49), doughnut mutants (D1 and D4), walnut mutants (W4 and W7) and frilly mutants (F2 and F4) were compared to their parent strain pK233.

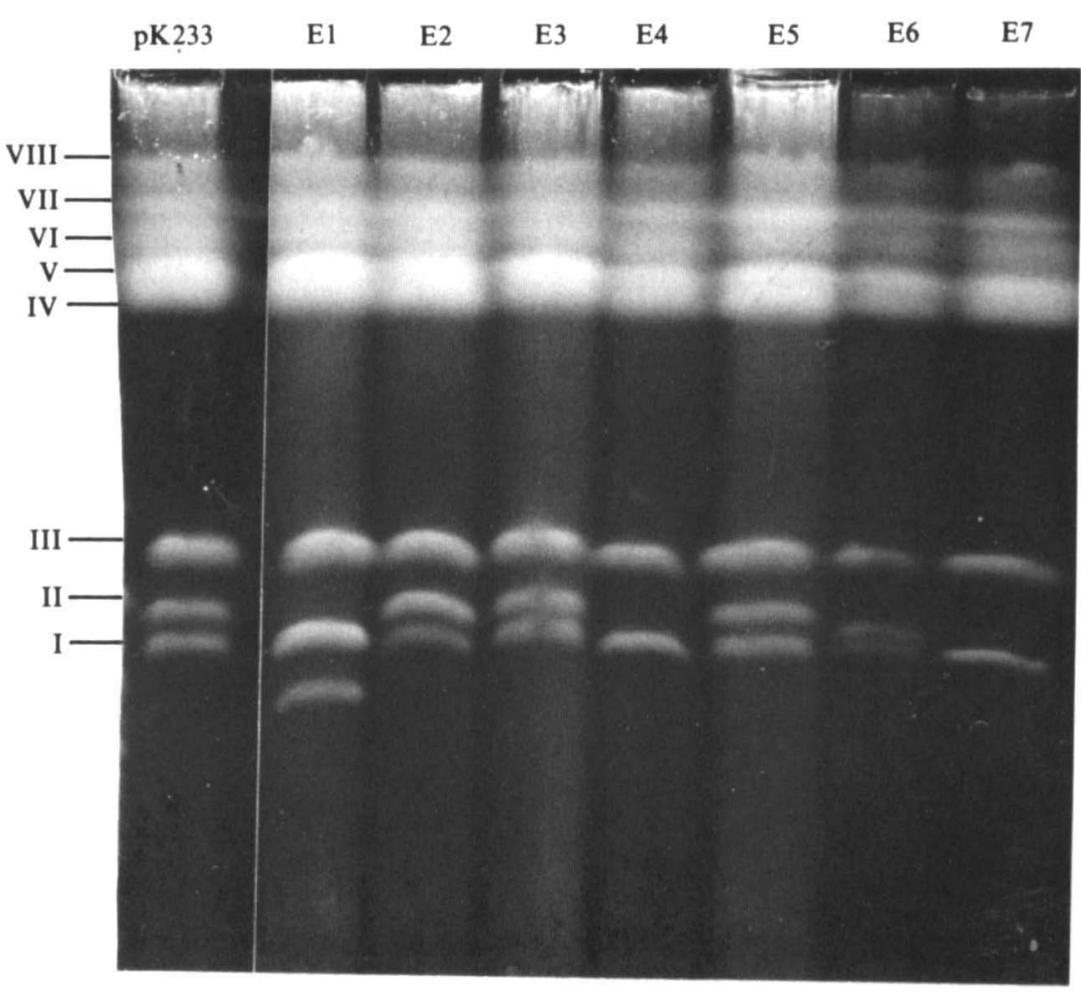


times longer than the former type. Long pseudohyphal cells were rarely detected in this case. Thus, the occurrence of filamentous pseudohyphal cells may be correlated to the wrinkled phenotype of colonies, as with the walnut mutants. In the frilly mutants, the colonies grown on 1/2YPD or YPBG plates contained pseudohyphal cells, together with normal yeast cells (data not shown) and were indistinguishable in cytological features from doughnut mutants.

In the case of the echinoid mutants, true hyphal cells were observed together with both normal-looking yeast cells and pseudohyphal ones (Fig. 2e). Branching was also observed. The terminal cell of true hyphae in these mutants was cylindrical and typically longer than the immediately preceding cell (Fig. $2 e-h$ ). The frequency of occurrence of these true hyphal cells was overwhelmingly higher than that of apparently normal-shaped yeast cells in the colony grown on 1/2YPD or YPBG plates. Hyphal germination from either yeast cells or pseudohyphal cells was also seen (Fig. $2 f, g$ ). When an echinoid mutant was grown on YPBO plates, the frequency of the true hyphal cells decreased in the doughnut-like colonies and pseudohyphal cells predominated.

\section{Chromosomal DNA banding patterns of colony- morphology mutants}

Chromosomal DNA of $C$. tropicalis pK233 was separated by PFGE into at least eight distinct bands (Fig. 3). The banding pattern was the same irrespective of the carbon source used for growth. Three bands around $1 \mathrm{Mbp}$ in size, and five bands around $2 \mathrm{Mbp}$, were separated. Individual chromosome-sized DNA bands were numbered I to VIII in ascending molecular size. Their apparent molecular sizes were estimated by using the chromosomal bands of $C$. albicans $\mathrm{FC} 18$ (Magee $e t$ al., 1988) and $S$. cerevisiae $\mathrm{X} 2180-1 \mathrm{~A}$ as standards.

The estimated sizes were $1.0 \mathrm{Mbp}$ (band I), $1.2 \mathrm{Mbp}$ (band II), 1.3 Mbp (band III), 2.1 Mbp (band IV), $2.2 \mathrm{Mbp}$ (band V), 2.4 Mbp (band VI), 2.6 Mbp (band VII) and $2.8 \mathrm{Mbp}$ (band VIII). Bands IV and V were close to each other and had enhanced staining compared to the other bands, suggesting that each was in turn composed of two bands. Therefore, the apparent total genome size was calculated to be approximately $19.9 \mathrm{Mbp}$, or $21 \mathrm{fg}$ per cell. Onthe other hand, chemical DNA determination with the parent strain gave the DNA content per cell to be 39-40 fg. The DNA content per cell of $S$. cerevisiae X2180-1a (haploid) and C. albicans $\mathrm{FC} 18$ was 18 and $38 \mathrm{fg}$, respectively. This suggests that the parent $C$. tropicalis strain may be diploid. In the colony-morphology mutants, some differences were detected in the banding patterns (Fig. 3). In frilly mutants (two representatives are shown in Fig. 3), band
VII of the parent was clearly absent in one example (F4). Instead, a new band appeared, the molecular size of which was between those of bands VII and VI, suggesting that band VII of the parent may be shortened in this, and possibly both, frilly mutants. No other additional bands were detected in these mutants. In the echinoid mutants, the difference was variable among isolates. One echinoid isolate (E26), had an additional band of $1.7 \mathrm{Mbp}$; another (E49), had a new $1.4 \mathrm{Mbp}$ band but had lost the parental band II. Furthermore in both isolates, the sizes of the sixth and the seventh bands, apparently equivalent to band VI and band VII of pK233, were less well resolved than the parental equivalents. In the echinoid isolates E1, E4, E6 and E7, the loss of band II was observed. In contrast, the echinoid isolates E2, E3 and E5 showed banding patterns that could not be distinguished from that of the parent strain.

No distinct difference was observed in the banding pattern of the doughnut or walnut mutants, compared to that of the parent.

Although the colony-morphology phenotype was different when glucose or oleic acid was used as carbon source, no significant difference was detected in the chromosomal banding patterns of any of the mutants grown in medium containing the different carbon sources.

\section{Discussion}

The colony-morphology mutants described here were isolated after UV-irradiation of low lethal effect $(20 \%$ or less). Considering that the DNA content of this organism is similar to $S$. cerevisiae diploid and many $C$. albicans strains, and that heterozygosity of naturally occurring auxotrophic markers is found (unpublished data), this organism cannot be haploid. The mutants isolated here are probably produced after chromosomal rearrangment (here, the term is interpreted in a broad sense, including mitotic crossing-over) accompanying segregation of naturally occurring heterozygous mutations, as has been shown in C. albicans for auxotrophic mutants (Whelan et al., 1980; Whelan \& Magee, 1981), as well as for morphological mutants (Pomes et al., 1985). The PFGE banding pattern of the karyotype of colony-morphology mutants described here indicates that the size of chromosomal DNA was changed in some of the mutants. This suggests that the chromosomal rearrangment includes mechanisms other than reciprocal crossingover. In the frilly mutants, the sizes of the bands apparently equivalent to band VI and band VII of the parent strain were variable among isolates. Ribosomal RNA genes have been assigned to band VI and band VII 
by hybridization experiments using the cloned rDNA genes as a probe (T. Kamiryo, personal communication). Repeated genes such as rDNA genes may contribute to high-frequency chromosomal rearrangement (reviewed by Petes \& Hill, 1988). Hybridization experiments using cloned genes from $C$. tropicalis are in progress.

Variation of colony morphology in the mutants may be explained to some degree by the cytological features of the constituent cells. A wrinkled phenotype correlates with the presence of pseudohyphal cells in chains, and the echinoid colony reflects the presence of aerial hyphae which are composed of filamentous true hyphae. However, it remains unknown how these and the other types of constituent cells assemble themselves to make various textures of individual colonies. No cytological difference can presently explain the differences in colony morphology between walnut, frilly and doughnut mutants.

The problem of colony-morphology phenotypes has two aspects, namely physiological and genetic variations. Both the variation of colony morphology with increase of incubation time and the variation with different medium composition, described in this paper, would belong to the former type.

True hyphae appeared in the echinoid mutant E49. Structures resembling germ-tubes, formation of which has been described as one of the important factors discriminating $C$. albicans from $C$. tropicalis (McGinnis, 1980), were also observed in this mutant (Fig. $2 f, g$ ). This suggests that the ability to produce germ-tube-like structures is present in the genome of $C$. tropicalis. The wrinkled texture of colonies correlating with the presence of filamentous pseudohyphal cells has also been found in morphological mutants of C. albicans (Gil et al., 1988; Rustchenko-Bulgac et al., 1990; Slutsky et al., 1987). Interspecific complementation analysis by protoplast fusion between this organism and $C$. albicans will provide more information on these problems, as has been reported for adenine auxotrophs (Corner \& Poulter, 1989).

This investigation was supported by Grants-in-Aid for Scientific Research from the Ministry of Education, Science and Culture of Japan (no. 01480018). We thank T. Kamihara (Kyoto University,
Kyoto, Japan) and T. Kamiryo (Hiroshima University, Hiroshima, Japan) for providing informations and useful discussions.

\section{References}

Burton, K. (1956). A study of the conditions and mechanisms of the diphenylamine reaction for the colorimetric estimation of deoxyribonucleic acid. Biochemical Journal 62, 315-323.

CARLE, G. F. \& Olson, M. V. (1984). Separation of chromosomal DNA molecules from yeast by orthogonal-field-alternation gel electrophoresis. Nucleic Acids Research 12, 5647-5664.

CORNER, B. E. \& POULTER, R. T. M. (1989). Interspecific complementation analysis by protoplast fusion of Candida tropicalis and Candida albicans adenine auxotrophs. Journal of Bacteriology 171, 3586-3589.

Gil, C., Pomes, R. \& Nombela, C. (1988). A complementation analysis by parasexual recombination of Candida albicans morphological mutants. Journal of General Microbiology 134, 1587-1595.

KamiRyo, T. \& OKAZAKI, K. (1984). High-level expression and molecular cloning of genes encoding Candida tropicalis peroxisomal proteins. Molecular and Cellular Biology 4, 2136-2141.

Kamiryo, T., Abe, M., Okazaki, K., Kato, S. \& Shimamoto, N. (1982). Absence of DNA in peroxisomes of Candida tropicalis. Journal of Bacteriology 152, 269-274.

McGinNIS, M. R. (1980). Laboratory Handbook of Medical Mycology. New York: Academic Press.

Magee, B. B., Koltin, Y., Gorman, J. A. \& Magee, P. T. (1988). Assignment of cloned genes to the seven electrophoretically separated Candida albicans chromosomes. Molecular and Cellular Biology 8, 4721-4726.

ODDs, F. C. (1987). Candida infections: an overview. CRC Critical Reviews in Microbiology 15, 1-5.

Petes, T. \& Hill, C. W. (1988). Recombination between repeated genes in microorganisms. Annual Review of Genetics 22, 147-168.

Pomes, R., Gil, C. \& Nombela, C. (1985). Genetic analysis of Candida albicans morphological mutants. Journal of General Microbiology 131, 2107-2113.

Rikkerink, E. H. A., Magee, B. B. \& Magee, P. T. (1988). Opaquewhite phenotype transition: a programmed morphological transition in Candida albicans. Journal of Bacteriology 170, 895-899.

Rustchenko-Bulgac, E., Sherman, F. \& Hicks, J. B. (1990) Chromosomal rearrangements associated with morphological mutants provide a means for genetic variation of Candida albicans. Journal of Bacteriology 172, 1276-1283.

SLUTSKY, B., BUFFo, J. \& Soll, D. R. (1985). High-frequency switching of colony morphology in Candida albicans. Science 230, 666-669.

STEWART, P. R. (1975). Analytical methods for yeasts. Methods in Cell Biology 12, 111-147.

Suzuki, T., Kobayashi, I., Kanbe, T. \& Tanaka, K. (1989). High frequency variation of colony morphology and chromosome reorganization in the pathogenic yeast Candida albicans. Journal of General Microbiology 135, 425-434.

Whelan, W. L. \& Magee, P. T. (1981). Natural heterozygosity in Candida albicans. Journal of Bacteriology 145, 896-903.

Whelan, W. L., Partridge, R. J. \& Magee, P. T. (1980). Heterozygosity and segregation in Candida albicans. Molecular and General Genetics 180, 107-113. 